\title{
The Role of Elasticity in Hardness Testing
}

\author{
Milton C. Shaw · Gabriel J. DeSalvo
}

(C) American Society for Metals 1972

The presently accepted theory for indentation hardness ignores the elastic stress field, and considers the material indented to behave in a rigid-plastic manner. This postulation provides a useful first approximation, but fails to explain several of the important characteristics of the process. A newly developed approach to plasticity makes it possible to include elastic effects, which play a major role when the indenter is blunt. After being reviewed, the new theory is applied to explain several of the phenomenae associated with practical hardness testing.

In the indentation hardness test, a blunt indenter that approximates a flat punch is forced into a plane surface. The size of the impression that remains after the indenter is removed is a measure of the hardness of the indented body. Hardness is usually expressed in units of pressure $\left(\mathrm{mg} / \mathrm{mm}^{2}\right.$ or psi), obtained by dividing the maximum applied load $(P)$ by the area of the indentation measured either over the surface of the indenter (Brinell hardness) or in the plane of the surface indented (Meyer hardness). In both the Brinell and Meyer tests, the indenter is a sphere, Fig. 1(a), and:

$$
\begin{aligned}
& H_{\mathrm{B}}=\text { Brinell hardness }=\frac{2 P}{\pi D^{2}\left(1-\sqrt{1-(2 a / D)^{2}}\right)} \\
& H_{\mathrm{M}}=\text { Meyer hardness }=\frac{P}{\pi a^{2}}
\end{aligned}
$$

Reprinted from Metals Engineering Quarterly, 12(2), 1-7, (1972), copyright $(\subset)$ American Society for Metals.

At the time of publication, Milton C. Shaw was professor and head, Department of Mechanical Engineering, Carnegie-Mellon University, Pittsburgh, Pa. Gabriel J. DeSalvo was senior engineer, Astronuclear Laboratory, Westinghouse Electric Corp., Pittsburgh, Pa.
Brinell and Meyer hardness values do not differ greatly, but the Meyer value is often preferred because of its simplicity and correspondence to the true mean stress over the area of contact.

Vickers, Fig. 1(b), and Knoop, Fig .1(c), indenters are blunt pyramids. Faces of the Vickers indenters are inclined at an angle of $136 \mathrm{deg}$, while the ridges of the Knoop indenter have angles of $130 \mathrm{deg}$ and $172.5 \mathrm{deg}$, respectively. Because the Knoop indenter penetrates only about half as deeply as the Vickers for the same load, it is frequently preferred for studies of superficial hardness. The Vickers hardness is the load divided by the contacting surface area, while the Knoop hardness is the load divided by the projected area, and hence corresponds to the Meyer value.

The hardness test is very easily conducted, but not so easily interpreted. Action beneath the indenter is complex, and must be understood if full use is to be made of hardness values. Basically, the hardness test is a measure of the resistance a material offers to plastic flow.

The simple compression test, Fig. 2, provides another measure of resistance to plastic flow that is more widely used in design analysis. If friction is kept to a low value on the die faces, a compression specimen will deform as shown by the dotted lines in Fig. 2, without barreling, and the uniaxial flow stress will be:

$\sigma_{1}=\frac{P}{A}$

where $A$ is the cross-sectional area of the specimen.

It is important to relate Meyer hardness to uniaxial flow stress, a term with which most engineers are accustomed. The plastic zone beneath a hardness indentation is surrounded by elastic material which acts to hinder plastic flow in a manner similar to the die surfaces in a closed die forging. In the simple compression test the entire specimen 


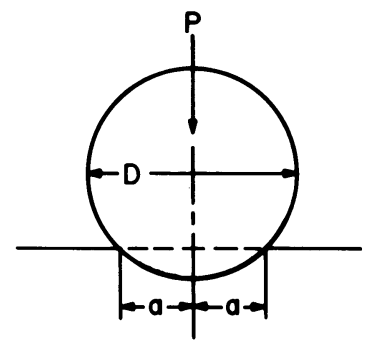

(a)

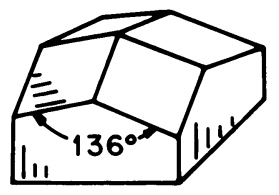

(b)

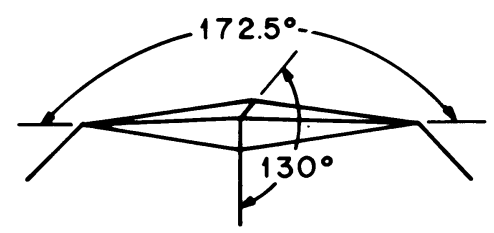

(c)

Fig. 1 Hardness indenters: (a) Brinell, (b) Vickers, and (c) Knoop

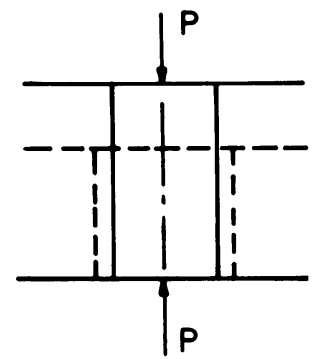

Fig. 2 Simple compression test

goes plastic, and there is no resistance to side flow because the specimen is surrounded by air. Therefore, a greater mean stress is required to cause plastic flow in the hardness test than in the simple compression test.

The relation between the Meyer hardness and the uniaxial flow stress may be expressed as follows:

$H_{\mathrm{M}}=C \sigma_{1}$

where $C$ is called the constraint factor for the hardness test. Experimentally, $C$ approximates three for the Brinell, Vickers, and Knoop hardness tests. A central problem in the theory of hardness is to explain the origin of constraint factor, $C$.

\section{Theory}

The currently adopted explanation of indentation hardness is given in terms of the so-called slip line field theory [1]. According to this theory, the material beneath a punch flows plastically over a region consistent with the material displaced by the punch. At all other points, the specimen is considered rigid. A suitable flow pattern need only be consistent from the point of view of velocities; when this is so, it is said to be a kinematically admissible solution.

The first kinematically admissible solution for a flat twodimensional punch is that shown in Fig. 3(a), due to Prandtl [2]. This flow pattern leads to a constraint factor of $1+\pi / 2=2.57$. Because conditions of equilibrium need not be satisfied in the slip line field approach, there are many possible solutions, each of which is an upper-bound solution. Another kinematically admissible flow pattern for the flat punch is that due to Hill [1], shown in Fig. 3(b). This leads to the same value of $C$ (2.57).

According to these theories, upward flow accounts for the material displaced by the punch. Constraint factor (C) may be termed a flow constraint from slip line field point of view.

When a large block of material having a grid applied to a central plane is loaded by a spherical indenter, flow patterns such as those shown in Fig. 4 are obtained. Study of these patterns reveals a plastic zone that passes through the edges of the punch, Fig. 4(c). There is no evidence of upward flow, and little resemblance to the plastic zones of Fig. 3.

The deformed grids of Fig. 4 clearly indicate an elasticplastic boundary, which has a shape resembling that of a line of constant maximum shear stress beneath a sphere

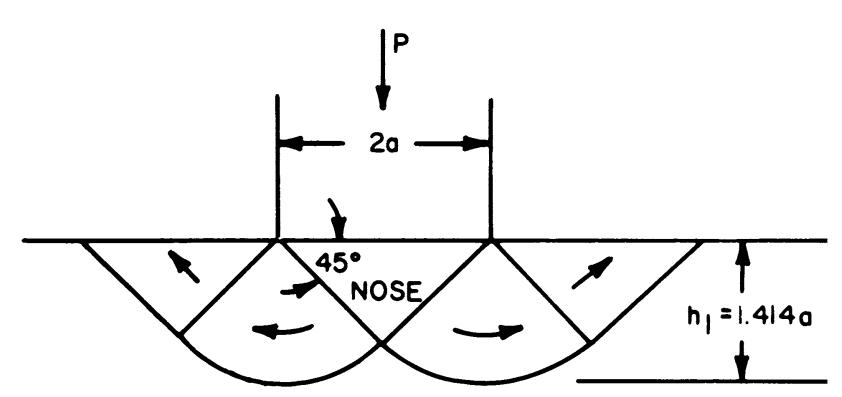

(a)

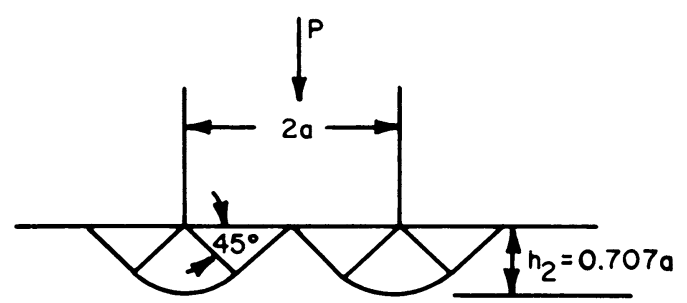

(b)

Fig. 3 Slip line field solutions for flat two-dimensional punch: (a) due to Prandtl [2] and (b) due to Hill [1] 
Fig. 4 Deformation of grid on meridional plane in a Brinell test: (a) plasticine, (b) mild steel, and (c) interpretation of (a) and (b) [3]

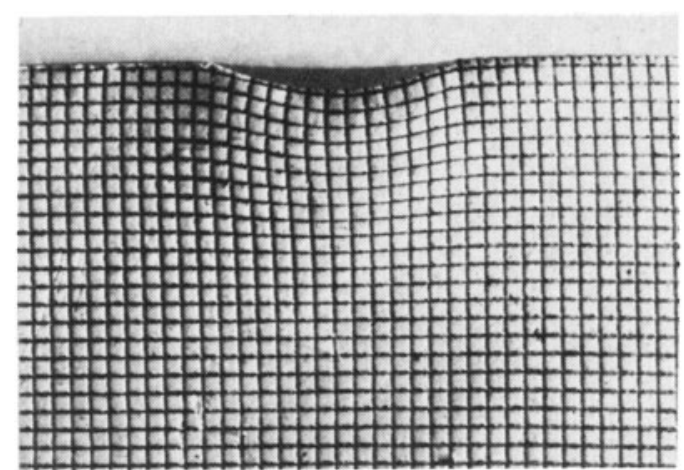

(a)
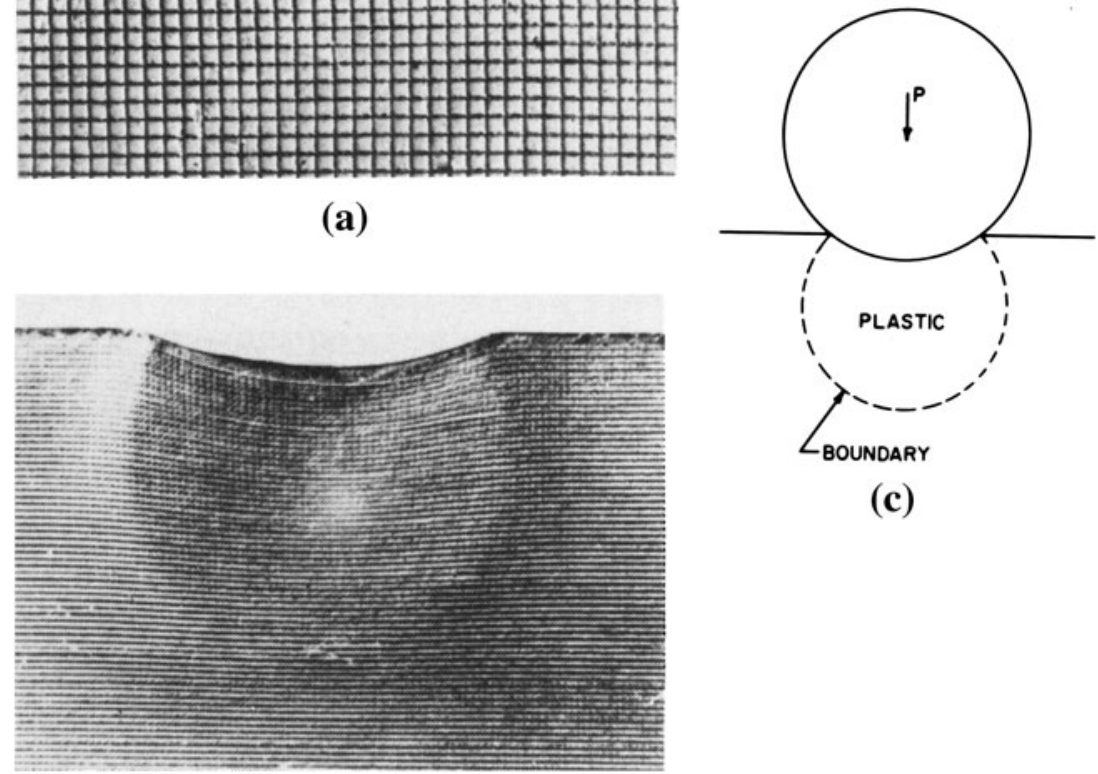

(c)

(b)

pressed against a flat surface. This elastic problem was first studied by Hertz. He found that the contact stress was distributed in a hemispherical pattern over the surface, and that lines of constant maximum shear stress were as shown in Fig. 5, where:

$M^{\prime}=\frac{\tau_{\max }}{\bar{p}}$

and

$\tau_{\max }=$ maximum shear stress

$\bar{p}=$ mean pressure on punch face (Meyer hardness)

In Fig. 5, the punch face is shown flat for simplicity, whereas in reality it is the surface of a large-radiused sphere. The elastic-plastic boundary of Fig. 4(c) closely resembles a line between $M^{\prime}=0.15$ and 0.20 .

A new approach to plasticity has recently been presented [3] in which the material is assumed to be plastic-elastic instead of plastic-rigid, as in all previously existing theory.

When applied to the indentation hardness problem, the new theory suggests that, if there is sufficient material beneath an indenter, the displaced material may be completely accounted for by the decrease in volume of the material elastically loaded in compression. There is then no

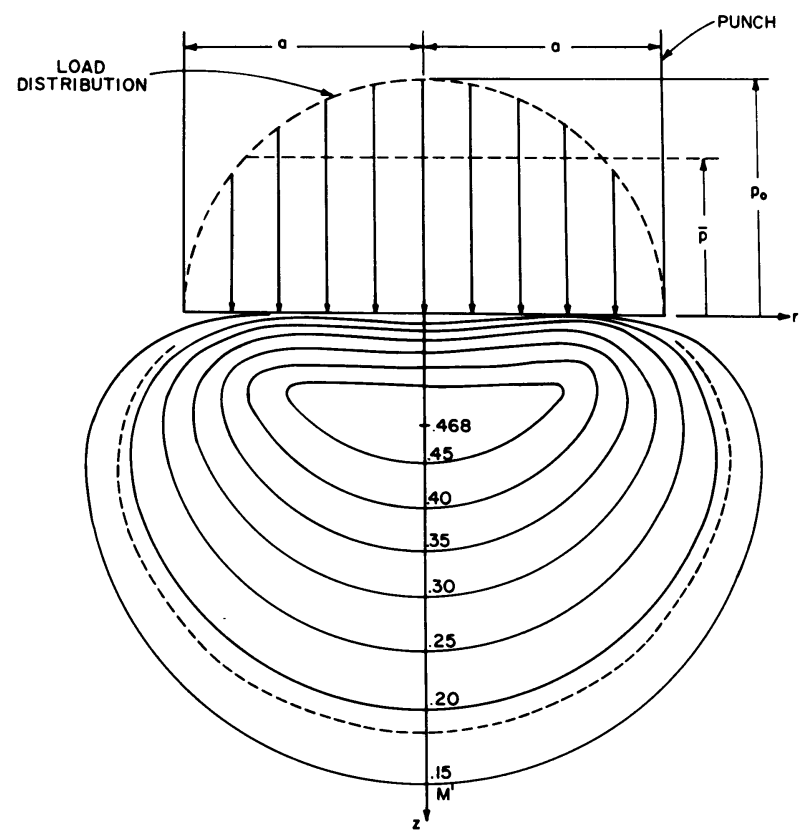

Fig. 5 Hertz lines of constant maximum shear stress for a frictionless spherical indenter: $M^{\prime}=\tau_{\max } / \bar{p}$

upward flow, as called for in all slip line field solutions. When the load is released, the impression remains (except for a small amount of elastic recovery) due to the residual 
elastic stresses which develop during plastic flow immediately beneath the indenter.

The constraint factor that arises in this way is termed an elastic constraint since the displaced volume is accommodated by an elastic decrease in volume (instead of by upward flow, as in the slip-line field approach).

Since there is no evidence of upward flow in Fig. 4(a) or 4(b), the constraint involved is of the elastic variety in both instances.

By the maximum shear theory of plasticity, one of the lines of Fig. 4 should correspond to the elastic-plastic boundary. The shear stress on this particular line should be $Y / 2$ (where $Y$ is the uniaxial flow stress of the material, as determined in an experiment such as that of Fig. 2). Reference 3 presents a procedure for predicting the line in Fig. 4 corresponding to the elastic-plastic boundary. When this procedure is applied, the curve corresponding to $M^{\prime}=0.177$ is found to represent the particular value. From Eq 5:

$M^{\prime}=\frac{\tau_{\max }}{\bar{p}}=\frac{Y}{2 \bar{p}}=0.177$

Or the corresponding value of constraint factor $C$ is:

$C=\frac{\bar{p}}{Y}=\frac{1}{2(0.177)}=2.82$

However, this constraint factor is referred to the area of the punch in actual contact during indentation (radius $a$ in Fig. 4) instead of the area of the plastic impression that remains after the test. These two areas will differ because the edge of the indenter is elastically loaded. When an adjustment is made for the elastically loaded area, the constraint factor based on the plastic impression is found to be $2.82 / 0.94=3.0$, which is in excellent agreement with experiment.

The amount of material required to enable an elastic constraint to be fully developed in a Brinell test of mild steel corresponds to a hemisphere of radius $(10)(2 a / D) D$; here, $2 a$ is the impression diameter and $D$, the ball diameter. The coefficient 10 is directly proportional to the ratio Young's Modulus/Meyer hardness $=E / \bar{p}$. For the standard Brinell test of mild steel $(3000 \mathrm{~kg}$ load on a $10 \mathrm{~mm}$ ball with $2 a / D=0.4)$, the impression should be surrounded by a sphere of material of radius $=10(0.4)(10)=40 \mathrm{~mm}$ (1.58 in.). If less material surrounds the impression there must be some upward flow. In fact, complete upward flow may be demonstrated by use of a thin layer of modeling clay (low $E$ ) on steel (very high $E$ ). The material beyond the plastic zone (steel) then has such a high Young's modulus relative to that of the clay that the plastic-rigid assumption holds, producing flow patterns almost identical in appearance to those in Fig. 3 [3].

Strain hardening and friction should have no influence on the value of a completely elastic constraint. However, these factors have an important influence on flow constraint.

In order that there be no upward flow, an indenter must be blunt (small cone angle). Increased friction will postpone upward flow as will an increased tendency for strain hardening. The effective cone angle for most indenters, however, is such that some upward flow results even when there is sufficient material surrounding the indenter to provide a full elastic constraint. Thus, most hardness tests correspond to a constraint that is predominantly elastic, but with a small flow component.

\section{Effective Cone Angle}

The effective cone angle is a very important variable in hardness testing. Dugdale [4] has extensively studied the constraint factor of indenters of different cone angle; his experimental results are summarized in Fig. 6. The constraint value was found to decrease markedly with decrease in semicone angle $(\theta)$ when a fully-worked specimen was indented with very low friction (line $A B$ ). However, the constraint increased with decrease in $\theta$ when an annealed material was indented with high friction (line $B C$ ). In Fig. 6, which clearly reveals the influence of friction and strain-hardening tendency, the horizontal dotted line shows the fully elastic constraint value from the new theory.

While most of the practical indenters are not conical, they may be assigned an effective half cone angle $(\theta)$. The effective cone angle for the sphere is the angle that the tangent to the sphere makes with the vertical at the edge of the indentation, Fig. 7(a). For the sphere:

$\theta=\cos ^{-1} \frac{2 a}{D}$

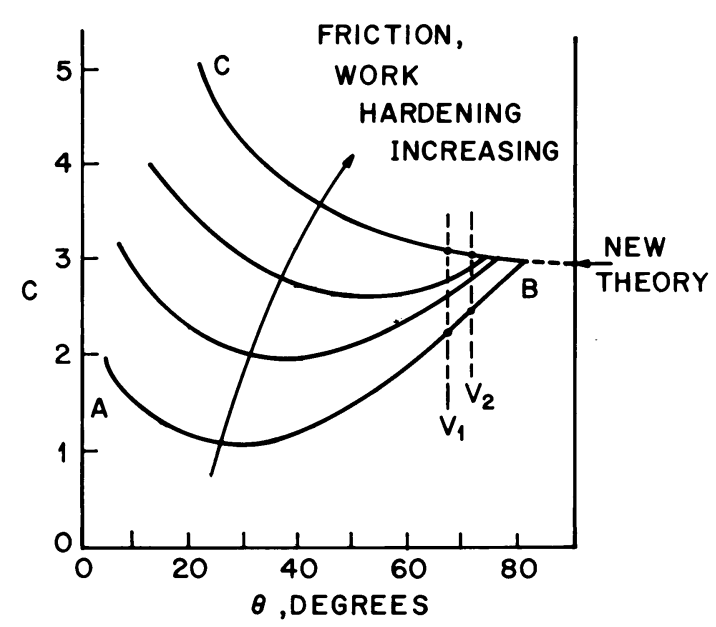

Fig. 6 Variation of constraint factor $C=\bar{p} / Y$ with cone semiangle $(\theta)$ for metals work-hardened to different degrees and with different amounts of friction on the surface of the indenter 
Fig. 7 Effective cone angles: (a) Brinell test, side elevation; (b) Vicker's indentation, plan view; and (c) Knoop indentation, plan view

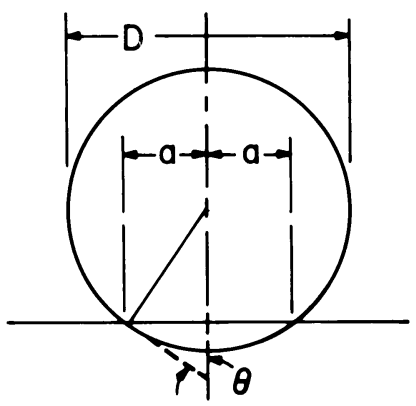

(a)

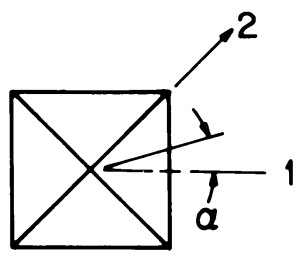

(b)

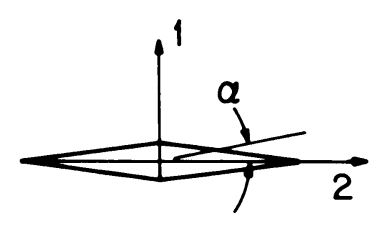

(c) when $2 a / D=0.4, \theta=66.4 \mathrm{deg}$.

For the Vicker's indenter, the cone angle $\theta$ is $136 / 2=$ $68 \mathrm{deg}$ for flow in Direction 1 in Fig. 7(b). For flow in a direction inclined at an angle $\alpha$ to Direction 1:

$\bar{\theta}=\tan ^{-1}\left(\frac{\tan 68}{\cos \alpha}\right)$

For Direction 2, this gives $\theta=72.18 \mathrm{deg}$. The mean value of $\theta$ may be obtained as follows:

$\theta=\frac{4}{\pi} \int_{0}^{\pi / 4} \tan ^{-1}\left(\frac{\tan 68}{\cos \alpha}\right) d \alpha=70.3 \mathrm{deg}$

For the Knoop indenter, Fig. 7(c) the cone angle $(\theta)$ is $130 / 2=65 \mathrm{deg}$ in Direction 1 , and 172.5/2 = 86.25 deg in Direction 2 . The mean value $\bar{\theta}$, found as above, is 72.12 deg.

These values are summarized as follows:

\begin{tabular}{llll}
\hline Indenter & $2 \bar{\theta}$ & $2 \theta_{\min }$ & $2 \theta_{\max }$ \\
\hline Sphere $(2 a / D=0.4)$ & 132.8 & $\ldots$ & $\ldots$ \\
Vicker's & 140.6 & 136 & 144.4 \\
Knoop & 144.2 & 130 & 172.5 \\
\hline
\end{tabular}

A sphere loaded such that $2 a / D=0.4$ is seen to be less blunt than either the Vicker's or Knoop indenters.

\section{Applications of Theory}

The new theory and the composite picture of Fig. 6 enable us to understand a number of phenomena associated with hardness testing that have not previously been fully explained. For example:

1. Why there is so little rise of material in the surface surrounding an indentation.

2. Why the apparent plastic strain in the hardness test is so low.

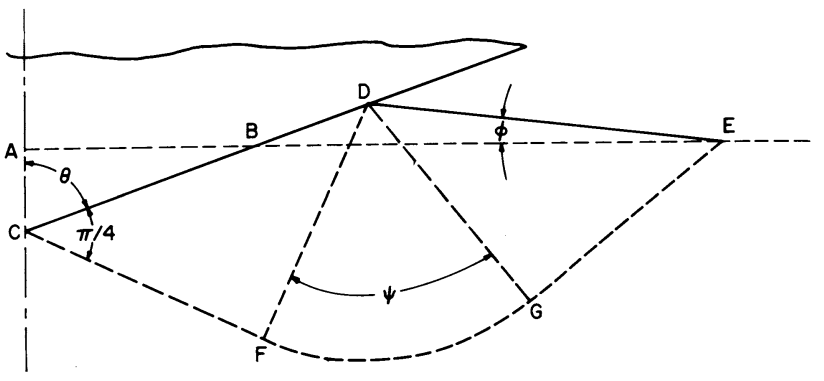

Fig. 8 Slip line field solution for frictionless two-dimensional wedge of 70 deg semiangle $(\theta)$

3. Origin of the "barrel" and "pin cushion" patterns in the Vicker's test.

4. Origin of the action of rising in fully worked material and sinking in annealed material at the lip of a hardness indentation.

5. Origin of the residual stresses in surfaces peened to improve the fatigue strength of parts.

6. Explanation of the "stainless indentation".

7. Meaning of the Meyer Exponent.

8. Why Brinell hardness values are sometimes too low for hard specimens, and why a spherical indenter approaches a fully-developed plastic state gradually.

Figure 8 shows the slip line field solution for a frictionless indenter with $2 \theta=140 \mathrm{deg}$. Since the material is assumed to behave in a plastic-rigid manner and there is no change in volume with plastic flow, the volume displaced $(A B C)$ must equal the volume in the lip $(B D E)$. In practice, however, the observed rise of material $(B D)$ will be a fraction of the value shown in Fig. 8 for a comparable hardness indenter (Vicker's with $2 \theta=140.6 \mathrm{deg}$ ).

Tabor [5] has empirically estimated the strain at the outer edge of a Brinell impression to be:

$\varepsilon \cong 0.2 \frac{2 a}{D}$

From Equation 6 this will be: 
$\varepsilon \cong 0.2 \cos \theta$

For a value of $\theta$ of $70 \mathrm{deg}, \varepsilon$ corresponds to 0.068 . From Fig. 7, the strain at the edge of the impression is:

$\varepsilon=\ln \frac{C D}{B C}=\ln 1.5=0.41$

which is about six times the experimental value.

The new theory explains both of these discrepancies. Because the bulk of the volume displaced is absorbed elastically, the volume displaced upward is a small fraction of that predicted by the plastic-rigid theory (Fig. 8).

When a Vicker's indenter is used on fully work-hardened material the impression will be barrel-shaped, as at $A$ in Fig. 9, but may have a pin-cushioned shape, as at $B$ in Fig. 9 when an annealed material is indented. The explanation for this phenomenon is found in Fig. 6. At the ridge of the Vicker's indenter, the effective cone angle is 144.4 $\mathrm{deg}$, but is only $136 \mathrm{deg}$ midway between the ridges. In Fig. 6, these values are indicated as $V_{2}$ and $V_{1}$, respectively. With annealed material, it is evident that it will take less force to displace material along the ridge $\left(V_{2}\right)$ than between the ridges $\left(V_{1}\right)$. As a consequence, this effect will lead to a flow pattern that is more extensive at the corners than between the corners (the pin cushion pattern). Similarly, if the material is fully cold-worked, line $A B$ in Fig. 6 will pertain instead of $B C$. Then it will take more force to displace material along the ridge $\left(V_{2}\right)$ than between ridges $\left(V_{1}\right)$. This effect, of course, leads to the barrel-shaped pattern.

Due to the cone angle being about $140 \mathrm{deg}$ for most practical indenters, there will be a small upward flow. This upward flow is accentuated if the elastic constraint is kept from developing due to insufficient material being present beneath the indenter. The upward flow will lie close to the surface of the indenter, and will "pile up" if the material is fully work-hardened and friction is low. For an annealed material, the upward flow will extend farther from the indenter, and will give the appearance of "sinking-in" at the indenter if friction is high.

The reason for this behavior is the same as that for the separation of curves $A B$ and $B C$ in Fig. 6. Figure 10 shows the slip line field for a frictionless indenter operating on a fully cold-worked specimen (solid lines); dotted lines

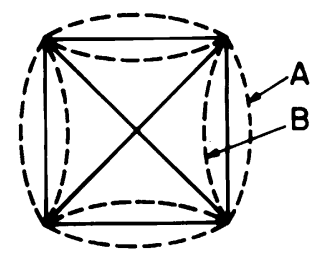

Fig. 9 Plan view of Vicker's indentation showing (a) barrel-shaped pattern, and (b) pin cushion-shaped pattern indicate the effects of considerable friction. If the material work hardens appreciably, the slip line pattern shifts in the direction of the dotted curve of Fig. 10. The constraint factor will be:

$C=1+\psi$

Since the value $\psi$ increases with either an increase in friction or an increased tendency to strain harden, the corresponding value of $C$ should increase as shown in Fig. 6. At the same time, the upward flow is loss localized near the indenter for annealed material, Fig. 10. With annealed material and high friction, the flow at the indenter will be retarded sufficiently to provide a sinking-in appearance.

If there were no elastic action, residual stresses would not arise. The large residual compressive stresses produced by shot peening to extend fatigue life are unexplained by the present theory of indentation hardness, which assumes the material to act in a plastic-rigid manner. The new plastic-elastic theory [3] enables the magnitude and extent of residual stresses to be estimated. The maximum residual compressive stress is estimated to be about $3.2 Y$ at the surface, and to fall to zero a distance $2.8 a$ beneath the surface of a spherical indenter (where $2 a$ is the diameter of the impression left in the surface, and $Y$ is the uniaxial flow stress).

The strainless indentation hardness is the value obtained by machining a cavity that precisely fits the indenter, and then loading until the first evidence of plastic flow is observed [6]. Alternatively [7], the test may also be performed by alternately indenting to the first sign of plastic action and annealing until a steady minimum value is reached. The so-called strainless hardness value obtained by either of these methods is about one-third the ordinary value of hardness. It is clearly the value of hardness in the absence of the residual stresses that arise during plastic flow in an ordinary hardness test. Alternately, it is the hardness value in the absence of elastic constraint. The strainless indentation hardness value should correspond to the uniaxial flow stress, which it does to an excellent approximation.

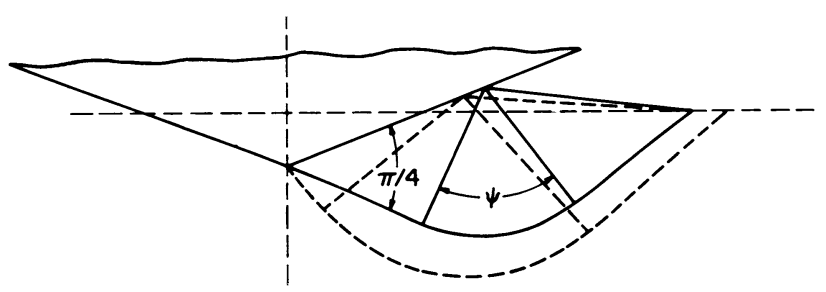

Fig. 10 Slip line field solution for two-dimensional wedge with $\theta=70 \mathrm{deg}$. Solid lines correspond to frictionless material and dashed lines to material with friction. The pattern for a frictionless indenter acting on annealed material will also resemble the dashed-line pattern 
It is interesting to note that lead tested slowly at room temperature should yield the strainless indentation hardness value because its strain recrystallization temperature is below room temperature. The same would be true for all metals when tested above their strain recrystallization temperatures. The hardness of a metal should decrease rather abruptly by a factor of approximately three when the temperature exceeds the strain recrystallization temperature. This effect should provide a convenient means for determining the strain recrystallization temperatures of cold-worked or annealed metals.

When the load $(P)$ on a spherical indenter is plotted against the diameter of the resulting impression $(2 a)$ on $\log -\log$ coordinates, a straight line is obtained, corresponding to the equation:

$P=A(2 a)^{n}$

The exponent $n$, called the Meyer index, is a measure of the tendency for the metal to strain harden. For a fully strainhardened metal, $n$ will be 2 ; for an annealed metal, $n$ will be about 2.5 . The quantity $A$ is a constant for a given ball size, but varies inversely as the ball diameter $(D)$ to the $n-2$ power. Thus, from $\mathrm{Eq} 12$ :

$\frac{P}{(2 a)^{2}}=A(2 a)^{n-2}=A^{\prime}\left(\frac{2 a}{D}\right)^{n-2}$

where $A^{\prime}$ is a constant for all ball sizes. From Eq 6 and the definition of Meyer hardness $\left(H_{\mathrm{M}}\right), \mathrm{Eq} 2$ :

$H_{\mathrm{M}}=\frac{4}{\pi} \frac{P}{(2 a)^{2}}=\frac{4}{\pi} A^{\prime}(\cos \theta)^{n-2}$

Equation 14 indicates that, for a fully work-hardened metal, the Meyer hardness $\left(H_{\mathrm{M}}\right)$ will be constant, since $n=2$. For an annealed metal, $H_{\mathrm{M}}$ for a sphere will increase with load, since $n>2$, and the cone angle $(\theta)$ will increase with load.

Because the wedge angle is constant for all loads in the Vicker's indenter, Eq 14 indicates there should be no change in the corresponding Meyer hardness with load for either an annealed or fully strain-hardened metal. This is found to be true experimentally; hence, the behavior of a Vicker's indenter is simpler to use than that of a sphere in this report. The Knoop indenter also has a constant mean effective cone angle for all loads, and gives the same hardness for all loads regardless of the state of strain hardening.

Other complications are associated with a spherical indenter. For a very light load (corresponding to $2 a / D \cong 0.1$ ), the constraint factor will be very low, slightly greater than one. The fully-developed constraint, three, will not be obtained until $(2 a / D)$ has reached about 0.4 . This effect is also believed due to elastic behavior, but of the indenter this time. For the full constraint of three to be obtained, the distribution of stress must be Hertzian, semicircular distribution, as shown in Fig. 5. According to Hertz, the semicircular stress pattern pertains only when a sphere deforms, under load, into another sphere of larger radius. When the applied load is small $(2 a / D \cong 0.1)$, it is conceivable that the indenter will deform as shown in Fig. 11, which in turn will cause the resulting constraint factor to be too low. Only after the sphere has penetrated a substantial distance into the specimen will the deformed indenter also be spherical, establishing the Hertzian distribution.

At any rate, a spherical indenter develops the full constraint of three gradually with load, and should not be used until $(2 a / D)$ has reached a value of about 0.4 for a spherical indenter made of steel. Since Vicker's and Knoop indenters show no such gradual approach to full plasticity, they can be used over a wider range of loads.

The fact that $(2 a / D)$ for fully-developed flow decreases as the value of Young's Modulus of a spherical indenter increases supports the theory that the phenomenon of gradual approach to full plasticity is associated with elastic deflection of the indenter. If a steel spherical indenter is used in a standard Brinell test $(3000 \mathrm{~kg}$ load, $10 \mathrm{~mm}$ diam ball) when the specimen hardness exceeds about $300 \mathrm{~kg} /$ $\mathrm{mm}^{2}$ (corresponding to $2 a / D=0.36$ ), the hardness value will be too low. A tungsten carbide sphere may be used for specimens with hardnesses of up to about $800 \mathrm{~kg} / \mathrm{mm}^{2}$; beyond this, it is advisable to use a Vicker's diamond.

\section{Practical Considerations}

The more blunt the indenter, the smaller will be the amount of upward flow. In the absence of upward flow, the hardness will be the same for an annealed and fully strainhardened specimen, and friction will play no role. Under such conditions, the hardness measured corresponds to the initial yield point of the material.

An indenter that is blunt enough to prevent upward flow produces an impression that is difficult to see and measure accurately. The effective cone angle must be decreased to a

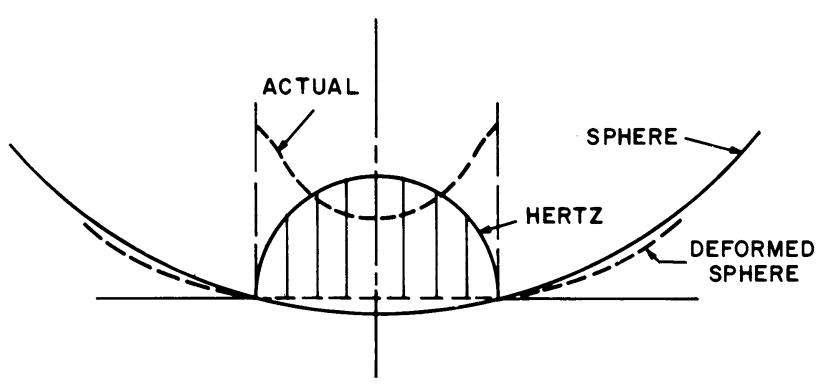

Fig. 11 Comparison of Hertzian and actual load distributions for a large sphere loaded over a small area 
value that approximates that for the Vicker's and Knoop indenters for accurate measurement. Presence of the ridges in Vicker's and Knoop indentations improves visibility, and hence accuracy of measurement. O'Neill [8] found that etched indenters also improved the accuracy of measurement, but had negligible influence on the size of the impression obtained.

With indenters having effective cone angles as small as those in the Vicker's and Knoop indenters, there will be a small amount of upward flow. The resulting constraint will still be essentially of elastic origin with a small flow component included. This flow component causes the hardness value to correspond to the flow stress at a small plastic strain (approximately $0.05 \mathrm{in}$./in.). It also alters the constraint factor upward or downward, depending upon whether the metal is fully strain-hardened, or annealed, and whether the indenter friction is high or low.

\section{Glasses and Polymers}

The foregoing discussion does not appear to hold for glasses and polymers, which appear to behave quite differently when indented.

While the possibility of glass flowing plastically in a hardness test has been questioned by some, Marsh [9] has presented extensive evidence supporting this phenomenon. For example, excellent microhardness impressions may be produced. Marsh finds constraint factors are much lower for glass than for metals. The ensuing discussion presents a possible explanation for this, employing the following notation:

$$
\begin{aligned}
& E=\text { Young's Modulus of Elasticity, psi } \\
& Y=\text { uniaxial flow stress (compression), psi } \\
& \bar{p}=\text { mean pressure on indenter, psi } \\
& v=\text { Poisson's ratio } \\
& C=\bar{p} / Y
\end{aligned}
$$

It appears as though solids should be divided into two classes from the point of view of hardness theory-those having high values of stiffness to flow stress $(E / Y)$, and those having low values of $E / Y$. When loaded by a blunt indenter, the materials having high values of $E / Y$ (metals) appear to develop a Hertzian stress distribution over the contact. Materials having low values of $E / Y$ (glasses and polymers), however, develop a uniform stress distribution over the contact area; in this sense, they behave as liquids. The value of $\bar{p} / Y$ (constraint factor, $C$ ) is a function of $E / Y$ for materials of low $E / Y$ (see Marsh [9], p. 424 and Fig., p. 425) but not for materials of high $E / Y$. The value of $\bar{p} / Y$ for low $E / Y$ materials (glass, polymers) is as follows to a very good approximation $(v=0.3)$, according to Marsh's theory for glasses and polymers: $\bar{p} / Y=0.28+0.60 \ln 0.7 E / Y$

The value given by this equation exceeds the constraint value for high $E / Y$ materials (3.0) when $E / Y$ is less than 133 , the critical value that distinguishes the low and high $E / Y$ regimes.

Steels will have values of $E / Y$ in the range 300 to 1000 , depending on hardness, while glasses and polymers will have values of $E / Y$ in the vicinity of 25 .

It would appear that the two types of behavior are related to a basic difference in atomic structure. Metals have long-range order, are extremely dense, and contain few imperfections relative to glasses and polymers. Glasses and polymers are capable of substantial densification when compressed whereas metals are relatively free of densification. It therefore appears as though the glasses and polymers behave as liquids in the vicinity of the applied load, and develop a stress distribution similar to that provided by a hydrostatically-loaded hemisphere. Metals, on the other hand, behave as true solids. The Hertz theory of elastic contact pertains, leading to a value of $\bar{p} / Y$ of three. Values of $\bar{p} / Y$ from $\mathrm{Eq} 15$ are greater than three when $E / Y$ exceeds 133 , but less than three when $E / Y$ is less than 133 .

Theories of elastic and flow constraint discussed in this paper apply only to materials having long-range order (metals). When considering materials of short-range order, which naturally have relatively low values of $E / Y(<133)$, the theory of Marsh pertains since it leads to lower values of $\bar{p} / Y$.

Acknowledgments A grant from the National Science Foundation was used in support of this study.

\section{References}

1. R. Hill, The Mathematical Theory of Plasticity (Oxford University Press, Oxford, 1950)

2. L. Prandtl, Nachr. Ges. Wiss. Gottingen, Math.-Phys. Kl., 74, (1920)

3. M.C. Shaw, G.J. DeSalvo, Trans. ASME 92, 469 (1970); Trans. ASME 92, 480 (1970)

4. D.S. Dugdale, Cone indentation experiments. J. Mech. Phys. Solids 2, 267 (1954); Experiments with pyramidal indenters-Part I. J. Mech. Phys. Solids 3, 197 (1955); Experiments with pyramidal indenters-Part II. J. Mech. Phys. Solids 3, 206 (1955)

5. D. Tabor, The Hardness of Metals (Clarendon, Oxford, 1951)

6. F.W. Harris, The hardness of the brasses, and some experiments on its measurement by means of a strainless indentation. J. Inst. Metals 28, 327 (1922)

7. E.G. Mahin, G.J. Foss, Absolute hardness. Trans. ASM 27, 337 (1939)

8. H. O'Neill, Carnegie Scholarsh. Mem. 15, 233 (1926)

9. D.H. Marsh, Plastic flow in glass. Proc. Roy. Soc. A279, 420 (1964) 